\title{
Effects of the Snl00 kVp Tube Voltage Mode on the Radiation Dose and Image Quality of Dual-Source Computed Tomography Pulmonary Angiography
}

This article was published in the following Dove Press journal:

International Journal of General Medicine

\author{
Wei-Guo Zhang ${ }^{1} * *$ \\ Jia-Ping $\mathrm{Liu}^{2, *}$ \\ Xiu-Qin Jia' \\ Ji-Yang Zhang \\ Xiang-Nan $\mathrm{Li}^{\prime}$ \\ Qi Yang (D)
}

'Department of Radiology, Beijing Chaoyang Hospital, Capital Medical University, Beijing, 100020, People's Republic of China; ${ }^{2}$ Department of Radiology, Beijing Xiongke Hospital, Capital Medical University, Beijing, I00049, People's Republic of China

*These authors contributed equally to this work
Correspondence: Qi Yang Department of Radiology, Beijing Chaoyang Hospital, Capital Medical University, No. 8 Gongti South Road, Chaoyang District, Beijing, 100020,

People's Republic of China

$\mathrm{Tel} / \mathrm{Fax}+86-85231928$

Email qiyang856@163.com

\begin{abstract}
Objective: This study aimed to investigate the effects of the $\mathrm{Sn} 100 \mathrm{kVp}$ tube voltage mode on the image quality and radiation dose of computed tomography pulmonary angiography (CTPA).

Methods: A total of 145 patients who underwent CTPA were randomly divided into five groups: control group (120 kVp, $150 \mathrm{mAs})$, test group A (Sn100 kVp, $270 \mathrm{mAs})$, test group B (120 kVp, $30 \mathrm{mAs})$, test group C (70 kVp, $150 \mathrm{mAs})$, and test group D (80 kVp, $70 \mathrm{mAs})$. After image post-processing, the image quality and radiation dose of each group were analyzed.
\end{abstract}

Results: The computed tomography values of images in the four test groups were more than $250 \mathrm{HU}$, which met the criteria for diagnosis. The signal-to-noise ratio and contrast-to-noise ratio of the images in the four test groups were lower than those in the control group. The radiation dose in each test group was lower than in the control group. The radiation dose was lowest in test group A.

Conclusion: The $\mathrm{Sn} 100 \mathrm{kVp}$ energy spectrum purification protocol can meet the requirements for clinical diagnosis, ensure image quality, and reduce the dose of radiation that patients receive.

Keywords: Sn100 kVp, CTPA, radiation dose, pulmonary embolism, dual-source CT

\section{Introduction}

Pulmonary embolism (PE) is a common clinical finding with a high death rate. $^{1-3}$ Computed tomography pulmonary angiography (CTPA) has become the first choice for the clinical diagnosis of pulmonary embolism due to its rapidity, non-invasive features, and high diagnostic accuracy. ${ }^{4}$ Given the widespread use of CTPA, more attention is being focused on problems associated with radiation.

Results of previous studies show that the doses of radiation from and contrast agent used in CTPA scanning can be greatly reduced by using dual-source, coarsepitch $\mathrm{CT}$ in combination with low tube voltage technology. ${ }^{5}$ Energy spectrum purification technology, $\mathrm{Sn} 100 \mathrm{kVp}$, has weak penetration, which is suitable for scanning the lungs. ${ }^{6,7}$ Therefore, this study discusses the image quality and dose of radiation from CTPA under the $\mathrm{Sn} 100 \mathrm{kVp}$ tube voltage mode to provide reference information for its future clinical application. 


\section{Data and Methods}

\section{Phantom Studies}

\section{Equipment and Phantom}

The scanning equipment was a third-generation, dual-source CT (SOMATOM Definition Force; Siemens Healthcare, Forchheim, Germany) with a detector array of 192 rows $\times$ $0.6 \mathrm{~mm}$. The phantom was a Catphan ${ }^{\circledR} 500$ from the American phantom laboratory, testing high-contrast resolution (spatial resolution), noise, and other modules, and using a spatial resolution module containing $1-21 \mathrm{LP} / \mathrm{cm}$ of precise line pairs. The Catphan ${ }^{\circledR} 500$ phantom was placed in the center of the CT frame for scanning.

\section{Scan Protocol}

The layer thickness was $0.75 \mathrm{~mm}$. The scanning field of view was the gross scanning field with a display field of view of $30 \mathrm{~cm}$, a matrix of $512 \times 512$, and a voxel display of $0.6 \mathrm{~mm}^{3}$. The pitch was 2.5 , the tube ball rotation time was $0.25 \mathrm{~s}$, and the convolution kernel was Br36. The scan parameters showed that the tube voltage was $120 \mathrm{kVp}, 80$ $\mathrm{kVp}, 70 \mathrm{kVp}$ and $\mathrm{Sn} 100 \mathrm{kVp}$, respectively. The tube current was 10-300 mA in $10 \mathrm{~mA}$ steps. Separate scans were performed using different tube voltage combinations. The high-resolution line logarithm and radiation dose of the combined images of all scanning parameters were observed and recorded after scanning.

\section{Image Analysis}

The GE picture archiving and communication system (PACS) was used for film reading. The spatial resolution, noise, and scanning dose of each scanning parameter were measured and recorded. The CTPA low-dose scan protocol was determined. The spatial resolution was represented by $\mathrm{LP} / \mathrm{cm}$ and observed with a window width of $0 \mathrm{HU}$ and a window position of 900-1000 HU. Adjacent line pairs with no adhesion under visual inspection, and the same line pair with no fractures, were taken as the evaluation standard. The spatial resolution was represented by calculating the maximum value of the line pair. Noise was represented by the standard deviation of the CT value in the region of interest (ROI). An ROI with an area of $100 \mathrm{~mm}^{2}$ was set in the center of the scanned image for measurement. The scanning dose was expressed as CT dose index volume $\left(\mathrm{CTDI}_{\mathrm{vol}}\right)$, which was automatically displayed by the CT machine after setting the scanning parameters. In the scan results, a spatial resolution of $5 \mathrm{LP} /$ $\mathrm{cm}$ was used as the standard. Scanning parameters satisfying these conditions were used in the clinical trials.

\section{Clinical Trial Standard}

According to WS 519-2019, when an image has a standard $^{8} \mathrm{CT}$ Dose Index Weighted $<50 \mathrm{mGy}$ and line pairs $>5.0 \mathrm{LP} / \mathrm{cm}$, it meets clinical diagnostic requirements. This was determined as the clinical trial group and compared with the conventional scanning parameters, $120 \mathrm{kVp}$ and $150 \mathrm{mAs}$.

\section{Clinical Studies General Data}

A total of 145 patients, who were treated in Beijing Chaoyang Hospital from November 2018 to December 2018 and suspected to have pulmonary embolism, were enrolled in the study for the CTPA examination. Patients were randomly divided into five groups, including, control group $(120 \mathrm{kVp}, 150 \mathrm{mAs})$, test group A (Sn100 kVp, $270 \mathrm{mAs})$, test group B (120 kVp, 30 $\mathrm{mAs})$, test group $\mathrm{C}(70 \mathrm{kVp}, 150 \mathrm{mAs})$, and test group $\mathrm{D}$ (80 kVp, $70 \mathrm{mAs})$, using a simple random grouping method.

Exclusion criteria: Pregnant or lactating women; patients with iodine contrast allergy; and patients with severe heart, liver, kidney, or respiratory dysfunction.

This study was approved by the Ethics Committee of Beijing Chaoyang Hospital (Ethical approval numbers: 2020-ke-26). This study was conducted in accordance with the Declaration of Helsinki. All patients signed an informed consent form before examination.

\section{Scan Method}

At the beginning of the scan, a breath-holding image of the chest was performed, with a scanning range from the thoracic entrance to the level of the diaphragm. The contrast agent used was Iopamiro $^{\circledR}$ (iohexol containing $370 \mathrm{mg} / \mathrm{mL}$ of iodine, Bracco Diagnostics), which was injected into the anterior elbow vein using a double-tube, high-pressure syringe. A mass injection of contrast agent tracking technology was used. A monitoring point was set in the main pulmonary artery. When the density of the monitoring point reached the threshold value, the scanning was automatically triggered. In the control group, $50 \mathrm{~mL}$ of the contrast agent was injected at a rate of $4.5 \mathrm{~mL} / \mathrm{s}$. When the density of the monitoring point reached $80 \mathrm{HU}$, the scan was automatically triggered, with a scan delay time of five seconds. In test groups A, B, C, and D, $30 \mathrm{~mL}$ of the contrast agent was injected at a rate of $4 \mathrm{~mL} / \mathrm{s}$. Then $30 \mathrm{~mL}$ of normal saline was injected at the same rate. When the density of the monitoring point reached 50 
$\mathrm{HU}$, the scan was automatically triggered, with a scan delay time of five seconds.

The scanning parameters of each group were determined according to the results of the phantom scanning. The tube voltage and reference milliampere per second were $\mathrm{Sn} 100 \mathrm{kVp}$ and $270 \mathrm{mAs}$ in test group $\mathrm{A}(\mathrm{n}=30)$, $120 \mathrm{kVp}$ and $30 \mathrm{mAs}$ in test group $\mathrm{B}(\mathrm{n}=28), 70 \mathrm{kVp}$ and $150 \mathrm{mAs}$ in test group $\mathrm{C}(\mathrm{n}=28), 80 \mathrm{kVp}$ and $70 \mathrm{mAs}$ in test group $\mathrm{D}(\mathrm{n}=29)$, and $120 \mathrm{kVp}$ and $150 \mathrm{mAs}$ in the control group $(n=30)$, respectively. The other scanning parameters were the same in each group, with a reconstruction layer thickness and layer spacing of $3 \mathrm{~mm}$ each. The image reconstruction algorithm was the advanced modeled iterative reconstruction (ADMIRE), with an iterative intensity of three, a convolution core value of Bv36, a reconstruction layer thickness of $1 \mathrm{~mm}$, and interlamellar spacing of $0.7 \mathrm{~mm}$.

\section{Image Post-Processing}

The thin-layer image data were transferred to the Siemens post-processing workstation (syngo.via VA30). Volume reproduction, maximum density projection, and multiplane reconstruction were performed on the lesion site using post-processing software to display the anatomy of the pulmonary arteries, local characteristics of the lesion, and other relevant image information accurately and intuitively.

\section{Image Quality Analysis}

Objective analysis: The measurement of the ROI in the objective quality assessment of images was carried out on a PACS workstation. It was completed by a physician with more than three years' experience in the Radiology Department. The CT values in the lumen of the pulmonary artery trunk, the pulmonary artery at the apical segment of the superior lobe of the right lung (the S1 segment), and the pulmonary artery at the posterior basal segment (the S6 segment) were measured using circular measurement tools and the CT values of the back muscles in the same layer as the main pulmonary artery. As far as possible, the ROI avoided the embolism site. If necessary, the ROI was measured in the lumen of the corresponding pulmonary arteries on the opposite side. The standard deviation of the air in the front of the thorax was measured as background noise. The signal-to-noise ratio (SNR) and contrast-tonoise ratio (CNR) of the images were calculated using the following formulae: SNR = intraluminal mean $\mathrm{CT}$ value $\div$ background noise and $\mathrm{CNR}=($ intraluminal mean
CT value - mean $\mathrm{CT}$ value of the back muscles $) \div$ background noise.

Subjective assessment: A three-grade rating system was used, ${ }^{3}$ in which Grade 1 meant that the image quality was excellent, the contrast agent was filling the pulmonary artery lumen well, and the diagnosis was made with full confidence. Grade 2 meant that the image quality was moderate, the contrast agent was filling the pulmonary artery lumen fairly well, and, while its CT value was lower than Grade 1, the image could meet diagnostic requirements. Grade 3 meant that the image quality was poor, and the contrast agent was sparse in the pulmonary artery lumen, so it was impossible to make a diagnosis. The image quality was scored independently by two doctors, each with more than three years' experience in the Radiology Department, using a double-blind method.

Radiation dose: The dose data in the dose report automatically generated by the $\mathrm{CT}$ machine, such as $\mathrm{CTDI}_{\mathrm{vol}}$ and dose-length production (DLP), were recorded. The effective dose (ED) per patient to complete this test was DLP $\times K$, where $\mathrm{K}$ refers to chest weight factor $(\mathrm{K}=$ $0.014)[\mathrm{mSv} \div(\mathrm{mGy} \cdot \mathrm{cm})]$.

\section{Statistical Analysis}

SPSS 22.0 statistical software was used. A one-way analysis of variance was used to compare the age, body mass index, radiation dose, CT value, and differences in SNR and CNR between the groups. A chi-squared test was used to compare the sex and PE distribution between the groups. A rank sum test was used to compare the subjective image quality scores between the groups. The $\mathrm{K}$ value was calculated to evaluate consistency $(\mathrm{K}=0.81-1.00$, excellent consistency; $\mathrm{K}=0.61-0.80$, good consistency; $\mathrm{K}=0.41-060$, average consistency; $\mathrm{K}<0.40$, poor consistency) of the image quality scores between the different observers. $\mathrm{P}<0.05$ was considered to be statistically significant.

\section{Results \\ Scanning Dose and Image Quality in Phantom Studies}

The combined spatial resolution was $6 \mathrm{LP} / \mathrm{cm}$ when tube voltage $=120 \mathrm{kVp}$ and tube current $\geq 150 \mathrm{mAs}$, tube voltage $=$ Sn $100 \mathrm{kVp}$ and tube current $\geq 270 \mathrm{mAs}$, tube voltage $=120$ $\mathrm{kVp}$ and tube current $\geq 30 \mathrm{mAs}$, tube voltage $=70 \mathrm{kVp}$ and tube current $\geq 150 \mathrm{mAs}$, and tube voltage $=80 \mathrm{kVp}$ and tube current $\geq 70 \mathrm{mAs}$. See Table 1 for details of scanning dose 
Table I Comparison of Scanning Dose and Image Quality of Different Combined Phantom Scans

\begin{tabular}{|l|c|c|c|c|c|}
\hline & $\mathbf{7 0 k v , ~ I 5 0 m A s ~}$ & $\mathbf{8 0 k v , 7 0 m A s}$ & Sn I00kv,270mAs & I 20kv,30mAs & I 20kv, I50mAs \\
\hline Spatial resolution & 6 & 6 & 6 & 6 & 6 \\
\hline Noise & 7.39 & 6.268 & 5.473 & 5.137 & 2.26 \\
\hline CTDI $_{\text {vol }}$ & 1.35 & 1.03 & 0.93 & 1.59 & 24.6 \\
\hline DLP & 20.8 & 16.7 & 14.4 & 0.34 & 122.9 \\
\hline ED & 0.28 & 0.23 & 0.20 & 1.72 \\
\hline
\end{tabular}

Abbreviations: $C T D I_{\text {vol, }}$ CT volume dose index; DLP, effective dose length product; $E D$, effective dose.

Table 2 Comparison of Basic Information of Subjects in Each Group

\begin{tabular}{|l|l|l|l|l|l|l|}
\hline & Control Group & Test Group A & Test Group B & Test Group C & Test Group D & $\boldsymbol{P}$ \\
\hline Age (years) & $62.83(35 \sim 84)$ & $66.06(35 \sim 83)$ & $62.67(32 \sim 85)$ & $56.89(32 \sim 78)$ & $65.79(34 \sim 91)$ & $0.8 \mathrm{I}$ \\
\hline Weight $(\mathrm{kg})$ & $66.13(43 \sim 10)$ & $64.33(47 \sim 80)$ & $63.85(42 \sim 92)$ & $70.60(48 \sim 100)$ & $67.03(42 \sim 90)$ & 0.37 \\
\hline Height $(\mathrm{m})$ & $1.65(1.55 \sim 1.80)$ & $1.65(1.50 \sim 1.78)$ & $1.6(1.50 \sim 1.78)$ & $1.67(1.51 \sim 1.82)$ & $1.66(1.57 \sim 1.82)$ & 0.87 \\
\hline BMI & $23.92 \pm 4.12$ & $23.50 \pm 2.5$ & $23.41 \pm 4.05$ & $25.23 \pm 3.76$ & $23.96 \pm 3.38$ & 0.35 \\
\hline
\end{tabular}

and spatial resolution results. In the phantom scanning, the scanning with a radiation dose of $\mathrm{Sn} 100 \mathrm{kVp}$ and $270 \mathrm{mAs}$ was $88 \%$ lower than that of $120 \mathrm{kVp}$ and $150 \mathrm{mAs}$. In addition, the spatial resolution of the image was maintained. At the same time, the noise was significantly lower than that of the low-dose groups, with $70 \mathrm{kVp}$ and $80 \mathrm{kVp}$, and slightly higher than that of the group with $120 \mathrm{kVp}$ and $30 \mathrm{mAs}$.

\section{Comparison of General Data}

In the clinical study, differences in sex, age, and body mass index (BMI) were not statistically significant $(\mathrm{P}>0.05)$ between the five groups. See Table 2 for the demographic information for each group.

\section{Comparison of Image Quality and Radiation Dose Between the Test Groups and the Control Group}

The difference in CT values for the main pulmonary artery and the S1 segment of the right lung was not significant between the control group and test groups $\mathrm{A}$ and $\mathrm{B}(\mathrm{P}>$ 0.05). The CT values of test groups $\mathrm{C}$ and $\mathrm{D}$ were significantly higher than those of the control group, and the difference was statistically significant $(\mathrm{P}<0.05)$. When the CT values of the S6 segment of the right lung were compared between the five groups, the difference was statistically significant $(\mathrm{P}<0.05)$ between test groups $\mathrm{A}$,
C, D, and the control group. Only test group B had the same CT value as the control group $(\mathrm{P}>0.05)$. The CT values of the images of the four test groups were more than $4 \mathrm{HU}$, which met the criteria of diagnosis. When the SNR and CNR were compared among the five groups, the difference was statistically significant $(\mathrm{P}<0.05)$ between the test groups A, B, C, D, and the control group. The $\mathrm{SNR}$ and CNR of the four test groups were lower than those of the control group. The differences in $\mathrm{CTDI}_{\mathrm{vol}}$, DLP, and ED values were not statistically significant $(\mathrm{P}<$ $0.05)$ between the test groups and the control group. The results showed that the radiation dose of each test group was lower than that of the control group when the image quality met the CTPA diagnosis; the radiation dose of test group A ( $\mathrm{Sn} 100 \mathrm{kVp})$ was the lowest, and the image met the diagnostic requirements. Details are shown in Table 3.

\section{Comparison of Subjective Image Quality and Consistency}

The subjective scores of images by two doctors were consistent $($ Kappa $=0.868, \mathrm{P}<0.05)$. Details are shown in Table 4.

\section{Discussion}

At present, CTPA is one of the main methods for the diagnosis of pulmonary embolism. The quality of the CTPA image is closely related to the enhancement of the 
Table 3 Comparison of Image Quality and Radiation Dose Between Test Groups and Control Group

\begin{tabular}{|c|c|c|c|c|c|c|c|}
\hline & Control Group & Test Group A & Test Group B & Test Group C & Test Group D & $x^{2}$ & $p$ \\
\hline $\begin{array}{l}\mathrm{CT} \text { values for the main pulmonary } \\
\text { artery }\end{array}$ & $\begin{array}{c}406.53 \\
(155.00 \sim 670.00)\end{array}$ & $\begin{array}{c}376.97 \\
(252.00 \sim 531.00)\end{array}$ & $\begin{array}{c}430.03 \\
(211.00 \sim 648.00)\end{array}$ & $\begin{array}{c}695.82 \\
(403.00 \sim 1078.00)^{*}\end{array}$ & $\begin{array}{c}600.24 \\
(351.00 \sim 1184.00)^{\#}\end{array}$ & 70.22 & 0.0001 \\
\hline $\begin{array}{l}\mathrm{CT} \text { values for the } \mathrm{SI} \text { segment of } \\
\text { the right lung }\end{array}$ & $\begin{array}{c}370.46 \\
(206.00 \sim 90.00)\end{array}$ & $\begin{array}{c}378.53 \\
(268.00 \sim 550.00)\end{array}$ & $\begin{array}{c}361.64 \\
(195.00 \sim 662.00)\end{array}$ & $\begin{array}{c}557.57 \\
(330.00 \sim 811.00)^{*}\end{array}$ & $\begin{array}{c}508.38 \\
(264.00 \sim 720.00)^{\#}\end{array}$ & 51.77 & 0.0001 \\
\hline $\begin{array}{l}\mathrm{CT} \text { values of the } \mathrm{S} 6 \text { segment of the } \\
\text { right lung }\end{array}$ & $\begin{array}{c}288.63 \\
(157.00 \sim 480.00)\end{array}$ & $\begin{array}{c}336.57^{\dagger} \\
(152.00 \sim 557.00)\end{array}$ & $\begin{array}{c}295.39 \\
(220.00 \sim 590.00)\end{array}$ & $\begin{array}{c}394.17 \\
(227.00 \sim 637.00)^{*}\end{array}$ & $\begin{array}{c}382.45 \\
(152.00 \sim 598.00)^{\#}\end{array}$ & 21.45 & 0.0001 \\
\hline SNR & $\begin{array}{c}70.90 \\
(4.00 \sim 215.00)\end{array}$ & $\begin{array}{c}36.07 \\
(17.00 \sim 58.00)^{\dagger}\end{array}$ & $\begin{array}{c}38.21 \\
(9.00 \sim 89.00)^{\ddagger}\end{array}$ & $\begin{array}{c}43.75 \\
(19.00 \sim 98.00)\end{array}$ & $\begin{array}{c}33.10 \\
(13.00 \sim 88.00)^{\#}\end{array}$ & 12.65 & 0.013 \\
\hline CNR & $\begin{array}{c}59.66 \\
(3.00 \sim 200.10)\end{array}$ & $\begin{array}{c}31.20 \\
(14.00 \sim 54.00)^{\dagger}\end{array}$ & $\begin{array}{c}32.25 \\
(6.00 \sim 78.00)^{\ddagger}\end{array}$ & $\begin{array}{c}38.96 \\
(16.00 \sim 89.00)\end{array}$ & $\begin{array}{c}28.97 \\
(10.00 \sim 78.00)^{\#}\end{array}$ & 11.79 & 0.019 \\
\hline $\mathrm{CTDI}_{\text {vol }}(\mathrm{mGy})^{\#}$ & $\begin{array}{c}7.99 \\
(7.97 \sim 8.00)\end{array}$ & $\begin{array}{c}0.92 \\
(0.74 \sim 0.95)^{\dagger}\end{array}$ & $\begin{array}{c}1.59 \\
(1.59 \sim 1.59)^{\ddagger}\end{array}$ & $\begin{array}{c}1.34 \\
(1.11 \sim 1.35)^{*}\end{array}$ & $\begin{array}{c}1.02 \\
(1.02 \sim 1.02)^{\#}\end{array}$ & 1141.70 & 0.0001 \\
\hline DLP (mGy cm) & $\begin{array}{c}244.82 \\
(181.20 \sim 289.20)\end{array}$ & $\begin{array}{c}29.81 \\
(18.9 .0 \sim 35.10)^{\dagger}\end{array}$ & $\begin{array}{c}45.94 \\
(34.30 \sim 62.00)^{\ddagger}\end{array}$ & $\begin{array}{c}39.22 \\
(30.20 \sim 50.50)^{*}\end{array}$ & $\begin{array}{c}31.09 \\
(23.90 \sim 35.80)^{\#}\end{array}$ & 119.16 & $<0.0001$ \\
\hline ED (mSv) & $\begin{array}{c}3.42 \\
(2.54 \sim 4.05)\end{array}$ & $\begin{array}{c}0.42 \\
(0.26 \sim 0.49)^{\dagger}\end{array}$ & $\begin{array}{c}0.64 \\
(0.48 \sim 0.87)^{\ddagger}\end{array}$ & $\begin{array}{c}0.55 \\
(0.42 \sim 0.71)^{*}\end{array}$ & $\begin{array}{c}0.44 \\
(0.33 \sim 0.50)^{\#}\end{array}$ & 119.10 & 0.0001 \\
\hline
\end{tabular}

Notes: ${ }^{\dagger}$ Represents a statistically significant difference between test group $A$ and the control group; ${ }^{\dagger}$ represents a statistically significant difference between test group $B$ and the control group; *represents a statistically significant difference between test group $C$ and the control group; ${ }^{*}$ represents a statistically significant difference between the test group $D$ and the control group.

Table 4 Comparison of Subjective Image Quality and Consistency

\begin{tabular}{|l|c|c|c|c|c|c|}
\hline & Control Group & Test Group A & Test Group B & Test Group C & Test Group D & P \\
\hline Consistency & $3.96(1.02 \sim 3.96)$ & $1.00(1.00 \sim 2.00)$ & $1.59(1.59 \sim 1.59)$ & $1.35(1.11 \sim 1.35)$ & $1.02(1.02 \sim 1.02)$ & $<0.0001$ \\
\hline Subjective Image Quality & $114.65(30.70 \sim 140.90)$ & $1.00(1.00 \sim 2.00)$ & $1.00(1.00 \sim 2.00)$ & $1.00(1.00 \sim 2.00)$ & $1.00(1.00 \sim 2.00)$ & $<0.0001$ \\
\hline
\end{tabular}

pulmonary artery. The more iodinated contrast agent that enters the pulmonary artery within a given time, the better the enhancement of the lumen and the better the image quality. However, an increase in the contrast agent may increase the incidence of contrast-induced nephropathy. ${ }^{9}$ The development of CT-related technology, such as coarse-pitch CT, increases the speed of data acquisition and shortens the best display time for the pulmonary artery, thereby reducing the amount of contrast agent needed. ${ }^{10}$

Bae et $\mathrm{al}^{11}$ reported that when the $\mathrm{CT}$ value of the pulmonary artery was greater than $250 \mathrm{HU}$ it would meet the diagnostic criteria for a pulmonary embolism. In this study, $30 \mathrm{~mL}$ of iodinated contrast agent was used in the test group. The CT value of the lumen of each pulmonary artery branch was greater than $250 \mathrm{HU}$. This is the same as the image quality achieved using $50 \mathrm{~mL}$ of contrast agent in the control group. Pulmonary artery CTA is usually performed by elbow vein administration. As it does not pass through the systemic circulation, it is not necessary to calculate the dosage of contrast agent according to body weight. ${ }^{12}$ This also provides a basis for the use of $30 \mathrm{~mL}$ of low-dose contrast agent.

CTPA requires a good contrast between the inside and the outside of the pulmonary artery. Usually, a $120 \mathrm{kVp}$ scan is recommended. ${ }^{13-15}$ Low-dose scanning includes various methods, such as reducing tube current, reducing tube voltage, coarse-pitch scanning, automatic tube voltage modulation, automatic tube current modulation, and the use of noise reduction filters and iterative reconstruction algorithms. Some studies ${ }^{8}$ suggest that the dose of radiation has a linear relationship with the tube current and an exponential relationship with the tube voltage. Therefore, reducing the tube voltage can decrease the dose of radiation more effectively than reducing the tube current. However, the research of Tang et $\mathrm{al}^{16}$ shows that reducing the tube voltage decreases the CNR of the image. 
In this study, the voltage of tube A in the test group was Sn100 kVp of energy spectrum purification, which was significantly lower than the $120 \mathrm{kVp}$ used in the control group. The radiation dose in the $\mathrm{Sn} 100 \mathrm{kVp}$ group was the lowest, when compared with the other test groups. At the same time, Siemens Force dual-source CT (the third-generation, dualsource CT) can selectively filter low-energy photons with a tin filter plate placed in front of the x-ray ball tube, which improves the $\mathrm{x}$-ray utilization rate and thus reduces the dose absorbed by the human body. ${ }^{10}$ This can better separate the low $\mathrm{kVp}$ and high $\mathrm{kVp}$ spectra, thereby improving the CNR of the image. After reconstruction with ADMIRE technology, image noise is gradually reduced with a gradual increase in reconstruction intensity. A gradual increase in the SNR and CNR of the image compensates for the increase in noise after the $\mathrm{kVp}$ reduction. This has the potential to significantly reduce noise and improve image quality.

\section{Conclusions}

In the present study, the Sn100 kVp energy spectrum purification protocol was used for adult CTPA. The results revealed that the $\mathrm{CTDI}_{\mathrm{vol}}$, DLP, and ED were significantly lower in test group A than in the scanning protocols of the other four groups $(\mathrm{P}<0.05)$. This suggests that energy spectrum purification technology can significantly reduce the dose of radiation absorbed by the human body. In CTPA, using the Sn100 kVp energy spectrum purification protocol can meet the needs of clinical diagnosis, ensure image quality, and reduce the dose of radiation that patients receive.

\section{Ethics Statement}

Ethical approval numbers: 2020-ke-26.

\section{Funding}

National Natural Science Foundation of China (62041601).

\section{Disclosure}

The authors report no conflicts of interest in this work.

\section{References}

1. Essien EO, Rali P, Mathai SC. Pulmonary embolism. Med Clin North Am. 2019;103(3):549-564. doi:10.1016/j.mcna.2018.12.013
2. Rali PM, Criner GJ. Submassive pulmonary embolism. Am J Respir Crit Care Med. 2018;198(5):588-598. doi:10.1164/rccm.201711-2302CI

3. Wang SY, Gao JB, Liu J, et al. Application of iterative model reconstruction in pulmonary artery "Double Low" imaging. J Pract Radiol. 2016;32(12):1940-1944.

4. Stamm JA, Wood KE. Too much of a good thing: appropriate CTPA use in the diagnosis of PE. $J$ Hosp Med. 2018;13(1):67-68. doi: $10.12788 / \mathrm{jhm} .2901$

5. Nie P, Wang X, Cheng Z, Ji X, Duan Y, Chen J. Accuracy, image quality and radiation dose comparison of high-pitch spiral and sequential acquisition on 128-slice dual-source CT angiography in children with congenital heart disease. Eur Radiol. 2012;22 (10):2057-2066. doi:10.1007/s00330-012-2479-1

6. Haubenreisser H, Meyer M, Sudarski S, Allmendinger T, Schoenberg SO, Henzler T. Unenhanced third-generation dual-source chest $\mathrm{CT}$ using a tin filter for spectral shaping at 100kVp. Eur J Radiol. 2015;84(8):1608-1613. doi:10.1016/j. ejrad.2015.04.018

7. Suntharalingam S, Allmendinger T, Blex S, et al. Spectral beam shaping in unenhanced chest CT examinations: a Phantom Study on dose reduction and image quality. Acad Radiol. 2018;25(2):153-158. doi:10.1016/j.acra.2017.08.011

8. Du QN, Sui X, Song W, et al. Third generation dual source CT large pitch $70 \mathrm{kVp}$ tube voltage $15 \mathrm{~mL}$ contrast dosage pattern for CT pulmonary artery imaging. Zhongguo Yi Хие Кe Хие Yuan Xие Bao. 2016;39(1):27-33.

9. Gulina A, Pan CX, Liu WY, et al. Preliminary study of energy spectral CT imaging combined with low-contrast agent for coronary CT angiography. Chin J Radiol. 2014;48(10):805-810.

10. Chen GZ, Zhang LJ, Schoepf UJ, et al. Radiation dose and image quality of $70 \mathrm{kVp}$ cerebral $\mathrm{CT}$ angiography with optimized sinogram-affirmed iterative reconstruction: comparison with 120 kVp cerebral CT angiography. Eur Radiol. 2015;25(5):1453-1463. doi:10.1007/s00330-014-3533-y

11. Bae KT, Tao C, Gürel S, et al. Effect of patient weight and scanning duration on contrast enhancement during pulmonary multidetector CT angiography. Radiology. 2007;242(2):582-589. doi:10.1148/ radiol.2422052132

12. He L, Wan ZG, Yin X, et al. Preliminary study of low concentration low dose contrast gemstone energy spectroscopy CT pulmonary artery imaging. Radiol Pract. 2016;31(1):59-63.

13. Davis A, Wolf TJ, Foster ER. Complex Task Performance Assessment (CTPA) and functional cognition in people with Parkinson's disease. Am J Occup Ther. 2019;73(5):7305205060p17305205060p9. doi:10.5014/ajot.2019.031492

14. Björkdahl P, Nyman U. Using 100- instead of $120-\mathrm{kVp}$ computed tomography to diagnose pulmonary embolism almost halves the radiation dose with preserved diagnostic quality. Acta Radiol. 2010;51(3):260-270. doi:10.3109/02841850903505222

15. Braun FM, Johnson TR, Sommer WH, Thierfelder KM, Meinel FG. Chest CT using spectral filtration: radiation dose, image quality, and spectrum of clinical utility. Eur Radiol. 2015;25(6):1598-1606. doi:10.1007/s00330-014-3559-1

16. Tang K, Wang L, Li R, Lin J, Zheng X, Cao G. Effect of low tube voltage on image quality, radiation dose, and low-contrast detectability at abdominal multidetector CT: phantom study. J Biomed Biotechnol. 2012;2012:130169. doi:10.1155/2012/130169 
International Journal of General Medicine

Dovepress

\section{Publish your work in this journal}

The International Journal of General Medicine is an international, peer-reviewed open-access journal that focuses on general and internal medicine, pathogenesis, epidemiology, diagnosis, monitoring and treatment protocols. The journal is characterized by the rapid reporting of reviews, original research and clinical studies across all disease areas. The manuscript management system is completely online and includes a very quick and fair peer-review system, which is all easy to use. Visit http://www.dovepress.com/ testimonials.php to read real quotes from published authors.

Submit your manuscript here: https://www.dovepress.com/international-journal-of-general-medicine-journal 